\title{
Estudo etnobotânico de plantas medicinais comercializadas no mercado público de Iguatu-Ceará, Brasil
}

\author{
Ethnobotanical study of medicinal plants sold in the public market of Iguatu-Ceará, Brazil \\ Estudio etnobotánico de plantas medicinales vendidas en el mercado público de Iguatu-Ceará,
} Brasil

Recebido: 26/04/2021 | Revisado: 04/05/2021 | Aceito: 09/05/2021 | Publicado: 23/05/2021

\author{
Antonio Carlos Nogueira Sobrinho \\ ORCID: https://orcid.org/0000-0002-9921-3350 \\ Universidade Estadual do Ceará, Brasil \\ E-mail: caiosobrinho@yahoo.com.br \\ Jéssica Alves Nunes \\ ORCID: https://orcid.org/0000-0002-6179-302X \\ Universidade Estadual do Ceará, Brasil \\ E-mail: alves.nunes@aluno.uece.br \\ Raquel Uchoa de Souza \\ ORCID: https://orcid.org/0000-0002-0737-4393 \\ Universidade Estadual do Ceará, Brasil \\ E-mail: raquel.uchoa@aluno.uece.br \\ Leonardo da Silva Lucena \\ ORCID: https://orcid.org/0000-0002-8984-4132 \\ Universidade Estadual do Ceará, Brasil \\ E-mail: leonardo.lucena@ aluno.uece.br \\ Francisco Bruno Gabriel da Silva \\ ORCID: https://orcid.org/0000-0002-3207-4472 \\ Universidade Estadual do Ceará, Brasil \\ E-mail: bruno.gabriel@ aluno.uece.br \\ Davi de Figueiredo \\ ORCID: https://orcid.org/0000-0003-2207-3297 \\ Universidade Estadual do Ceará, Brasil \\ E-mail: davi.figueiredo@aluno.uece.br
}

\begin{abstract}
Resumo
As plantas com ação medicinal são recursos de primeira escolha para muitos grupos populacionais com acesso restrito aos programas governamentais de saúde pública. Tais plantas são facilmente encontradas e comercializadas em feiras livres e em mercados públicos de forma in natura ou seca, sendo considerada uma prática cultural. Este trabalho teve como objetivo identificar as plantas medicinais comercializadas em uma feira livre e no Mercado Público Municipal de Iguatu, no Estado do Ceará, Brasil, com o intuito de apresentar suas indicações terapêuticas, princípios ativos e meios de transmissão deste conhecimento pelos sujeitos, comerciantes e erveiros. Com fundamento na abordagem quali-quantitativa, fazendo uso de entrevistas semiestruturadas com comerciantes erveiros, foi realizado um levantamento das plantas medicinais utilizando a técnica de listagem livre. A partir da literatura especializada foi possível a identificação botânica das espécies comercializadas. Os resultados da pesquisa permitiram a identificação de 59 etnoespécies, pertencentes a 55 gêneros e distribuídas em 38 famílias botânicas, sendo Fabaceae e Lamiaceae as famílias mais representativas, ambas com 8,5\%, seguida de Asteraceae com 6,5\%. Em relação à parte vegetal mais utilizada destacou-se as cascas e caules $(27,42 \%)$ e sementes $(24,19 \%)$ e o modo de preparo predominante foi o chá $(98,3 \%)$. Dentre as espécies citadas pelos erveiros, $22 \%$ são utilizadas para tratar mais de um problema de saúde, enquanto 78\% possuem apenas uma indicação terapêutica. Quanto às condições sanitárias foram observadas algumas condições inadequadas de armazenamento de materiais vegetais para comercialização.
\end{abstract}

Palavras-chave: Etnomedicina; Etnobotânica; Plantas curativas; Saúde popular.

\begin{abstract}
Medicinal plants are resources of first choice for many population groups with restricted access to government public health programs. Such plants are easily found and sold in open-air markets and public markets in a fresh or dry way, being considered a cultural practice. This work aimed to identify the medicinal plants sold in an open market and in the Municipal Public Market of Iguatu, in the State of Ceará, Brazil, to present their therapeutic indications, active principles and means of transmission of this knowledge by herbs traders. Based on the qualitative and quantitative approach, making use of semi-structured interviews with herbs traders, a survey of medicinal plants was carried out
\end{abstract}


using the free listing technique. From the specialized literature, it was possible to identify the botanical species sold. The results of the research allowed the identification of 59 ethnospecies, belonging to 55 genera and distributed in 38 botanical families, with Fabaceae and Lamiaceae being the most representative families, both with $8.5 \%$, followed by Asteraceae with $6.5 \%$. Regarding the most used vegetable part, bark, and stems $(27.42 \%)$ and seeds $(24.19 \%)$ stood out and the predominant method of preparation was tea (98.3\%). Among the species cited by herbs traders, $22 \%$ are used to treat more than one health problem, while $78 \%$ have only one therapeutic indication. As for the sanitary conditions, some inadequate conditions of storage of plant materials for commercialization were observed.

Keywords: Ethnomedicine; Ethnobotany; Healing plants; Popular health.

\section{Resumen}

Las plantas medicinales son recursos de primera elección para muchos grupos de población con acceso restringido a los programas gubernamentales de salud pública. Estas plantas se encuentran y venden fácilmente en mercados abiertos de forma fresca o seca, siendo considerada una práctica cultural. Este trabajo tuvo como objetivo identificar las plantas medicinales vendidas en un mercado abierto y en el Mercado Público Municipal de Iguatu, en el Estado de Ceará, Brasil, con el fin de presentar sus indicaciones terapéuticas, principios activos y medios de transmisión de este conocimiento por parte de los comerciantes de hierbas. Sobre la base del enfoque cualitativo y cuantitativo, haciendo uso de entrevistas semiestructuradas con comerciantes de hierbas, se llevó a cabo una encuesta de plantas medicinales utilizando la técnica de listado libre. A partir de la literatura especializada, fue posible identificar las especies botánicas vendidas. Los resultados de la investigación permitieron identificar 59 etnoespecies, pertenecientes a 55 géneros y distribuidas en 38 familias botánicas, siendo Fabaceae y Lamiaceae las familias más representativas, ambas con $8.5 \%$, seguidas de Asteraceae con $6.5 \%$. En cuanto a la parte vegetal más utilizada, se destacaron corteza y tallos $(27,42 \%)$ y semillas $(24,19 \%)$ y el método de preparación predominante fue el té $(98,3 \%)$. Entre las especies citadas por los comerciantes, el $22 \%$ se utiliza para tratar más de un problema de salud, mientras que el $78 \%$ tiene una sola indicación terapéutica. En cuanto a las condiciones sanitarias, se observaron algunas condiciones inadecuadas de almacenamiento de materiales vegetales para su comercialización.

Palabras clave: Etnomedicina; Etnobotánica; Plantas curativas; Salud popular.

\section{Introdução}

As plantas medicinais representam um importante recurso nos cuidados com a saúde, de modo a contribuir significativamente na atenção primária à saúde. Dentro desse contexto, no Brasil, foi aprovada em 2006 a Política Nacional de Práticas Integrativas e Complementares (PNPIC), mediante a portaria $n^{\circ}$ 971, de 3 de maio de 2006, que inclui a fitoterapia como um serviço prestado no Sistema Único de Saúde - SUS (Brasil, 2006a). O Ministério da Saúde, por meio do Decreto ${ }^{\circ}$ 5.813, de 22 de junho de 2006, instituiu a Política Nacional de Plantas Medicinais e Fitoterápicos-PNPMF, eixo relevante da PNPIC, com o intuito de promover a população brasileira o acesso seguro na utilização de plantas medicinais e fitoterápicos (Brasil, 2006b). Conforme salientam Novaes et al. (2021), a inserção de estudos etnobotânicos na saúde pública se demonstra eficaz na promoção da valorização cultural local de uma comunidade, sendo possível o compartilhamento, por meio da oralidade, principalmente, de conhecimentos da medicina tradicional, que auxiliam na prevenção e tratamento de doenças, ou seja, na educação em saúde.

$\mathrm{Na}$ medicina popular, o uso de plantas com propriedades terapêuticas é uma prática bastante comum pelas camadas mais pobres da população, com menor cobertura de assistência médica, como as comunidades rurais que fazem desse recurso, muitas vezes, a única alternativa para o tratamento de doenças (Barreto \& Spanholi, 2019). A aquisição do conhecimento tradicional sobre o uso de plantas para fins medicinais, normalmente, ocorre de maneira empírica, através da verbalização, de geração em geração (Cerqueira et al., 2020). Contudo, fatores como o desinteresse dos jovens pela cultura popular, afastamento das tradições da agricultura familiar, migração para centros urbanos, têm contribuído para o declínio das tradições populares relacionadas à utilização das plantas medicinais (Couto, 2016).

A etnobotânica é a ciência que investiga os conhecimentos populares que o homem tem sobre o uso das plantas (Vásquez, Mendonça, Noda, 2014). Esse campo de estudo busca recuperar e preservar os saberes tradicionais das pessoas em relação às espécies, seus usos, manejos e relações com o ambiente (David \& Pasa, 2015). Estudos etnobotânicos podem ser desenvolvidos nos mais distintos espaços, como as feiras livres, por exemplo. O agrupamento de diversidade biológica e 
cultural, além do processo de troca de saberes que configuram esses locais, modificam as feiras em relevantes meios de pesquisa (Albuquerque et al., 2014).

As feiras e mercados públicos são espaços que centralizam grande pluralidade de plantas, utilizadas para fins alimentícios, medicinais e ornamentais, ao mesmo tempo em que possibilita a junção de vários atores sociais do sistema alimentar, dentre eles produtores e consumidores (Sales et al., 2011). Desta forma, esses são centros significativos para mensurar a variedade dessas plantas que são acessíveis à população, além da obtenção de conhecimentos sobre os usos populares ligados a essas plantas, que vem sendo apagados com o passar dos anos.

No Brasil, um país de dimensões continentais, há poucos estudos que relatam a importância e listam as espécies vegetais comercializadas em feiras livres e/ou mercados públicos, a destacar estudos no Rio de Janeiro (Azevedo \& Silva, 2006; Maioli-Azevedo \& Fonseca-Kruel, 2007; Bochner et al., 2012), em Cuiabá (Pinto et al., 2013) e no Estado do Pará (Lima, Coelho-Ferreira, Oliveira, 2011; Santos, Coelho-Ferreira, Lima, 2018). Na região nordeste, com padrões culturais, sociais e florísticos próprios, há relatos de identificação de plantas com ação medicinal e de práticas socioculturais em feitas livres e/ou mercados públicos em todas as regiões, com destaque para estudos em São Luís, Maranhão (Loch et al., 2020), no município de São Miguel, Rio Grande do Norte (Freitas et al., 2012), em Arapiraca, Alagoas (Lima, Nascimento, Silva, 2016) e no litoral piauiense, no município de Parnaíba (Santos et al., 2019). Todos esses trabalhos apontam para a necessidade do resgate e valorização dos conhecimentos populares, que assentam nos povos tradicionais e suas práticas socioculturais.

Diante desta perspectiva, o objetivo deste trabalho foi identificar as plantas medicinais comercializadas em uma feira livre e no mercado público municipal de Iguatu, estado do Ceará, além de suas indicações terapêuticas, princípios ativos e meios de transmissão deste conhecimento pelos comerciantes/erveiros.

\section{Metodologia}

O estado do Ceará situa-se no norte da Região Nordeste do Brasil $\left(05^{\circ} 05^{\prime} \mathrm{S}, 39^{\circ} 39^{\prime} \mathrm{O}\right)$, limitando-se ao norte com o Oceano Atlântico, ao leste com os estados do Rio Grande do Norte e Paraíba, a oeste com o estado do Piauí e ao sul com o estado de Pernambuco (IBGE, 2020a).

O local da pesquisa corresponde ao Mercado Público Municipal de Iguatu (latitude $06^{\circ} 21^{\prime} 34^{\prime \prime} \mathrm{S}$, longitude $39^{\circ} 17^{\prime}$ 55" O, altitude $218 \mathrm{~m}$ ), município localizado na região Centro-Sul do estado do Ceará, distante $380 \mathrm{Km}$ de sua capital, Fortaleza. A cidade de Iguatu possui uma população estimada em 103.074 habitantes (IBGE, 2020b), ocupando uma área territorial de 1.029,2 $\mathrm{km}^{2}$ e tendo densidade demográfica de 94,87 hab. $/ \mathrm{km}^{2}$ (IPECE, 2017).

O estudo foi realizado durante o mês de março de 2019, por meio de entrevistas semiestruturadas, com os comerciantes de plantas medicinais do mercado público e de uma feira livre que ocorre aos sábados no entorno do mercado público de Iguatu, o qual encontra-se situado mais precisamente na Av. Agenor Araújo S/N. A pesquisa foi desenvolvida em dias distintos nas semanas. Aos sábados, o fluxo de pessoas e a variedade de produtos comercializados no mercado é bem maior se comparado aos dias comuns da semana, atraindo, assim, várias pessoas da própria cidade e regiões circunvizinhas (Barbosa, 2019).

Para o levantamento das plantas medicinais utilizou-se a técnica de listagem livre, que se baseia em solicitar informações aos informantes sobre o objeto ou assunto que está sendo investigado. A técnica de listagem livre consiste em concepções que são mais importantes culturalmente e surgirão durante a listagem em sua ordem de importância para cada cultura (Albuquerque \& Lucena, 2004). Para manter o anonimato dos participantes da pesquisa, estes foram identificados como comerciante 1 a 5 .

Muitas das plantas foram adquiridas na forma comum de comercialização seca, o que impediu a coleta e herborização de algumas espécies. Contudo, foram realizados registros fotográficos e anotações em diários de campo de todas as plantas 
comercializadas para fins medicinais. A identificação botânica foi realizada por meio de consultas à literatura especializada, de modo a comparar os nomes vernaculares citados com os respectivos nomes científicos encontrados, além de comparação com o material testemunho depositado no Herbário Prisco Bezerra (EAC) da Universidade Federal do Ceará (UFC), quando necessário. A nomenclatura botânica utilizada encontra-se conforme a base de dados nacional Flora do Brasil 2020 (http://floradobrasil.jbrj.gov.br/), pertencente ao programa Reflora, e as bases de dados internacionais The Plant List (www.theplantlist.org) e World Flora Online (http://worldfloraonline.org/).

Para a identificação de cascas e raízes, recorreu-se a ajuda de especialistas, bem como fazendo o uso de identificação por meio de propriedades organolépticas do material, como aspecto, cor, odor e textura, identificação feita pela equipe de autores desse trabalho. Para Albuquerque et al. (2007), quando da realização de estudos com materiais vegetais secos e em formas comerciais, a coleta e herborização fica prejudicada, sendo útil o uso de identificação por consulta às bases de dados de identificação botânica, literatura especializada, bem como por procedimentos farmacobotânicos, como já realizados em outros estudos (Lima, Coelho-Ferreira, Oliveira, 2011; Williams, Witkowski, Balkwill, 2005). A lista de plantas selecionadas foi comparada com a Relação Nacional de Plantas Medicinais de Interesse ao SUS - RENISUS (Brasil, 2009), com o intuito de enfatizar a representatividade das espécies da RENISUS que são comercializadas no Mercado Municipal de Iguatu, assim como utilizar essa relação como parâmetro para as plantas medicinais com monografias já escritas.

\section{Resultados e Discussão}

A feira livre e o mercado público municipal, além de comercializarem plantas medicinais, apresentam setores específicos para a venda de peixes, hortifrúti, itens de vestuário, entre outros, de modo que os comerciantes se organizarem conforme o tipo de mercadoria que trabalham. O fluxo de pessoas nesses espaços ao longo da semana é variável, havendo uma maior quantidade de bancas aos sábados, devido diversos produtores irem ao centro urbano para a venda de seus produtos, ocasionando uma maior movimentação neste dia.

Havia apenas cinco feirantes que comercializavam plantas medicinais $(n=5)$, e quando questionados como ingressaram nesta área de venda de plantas medicinais, relataram ter sido instigados por familiares que repassaram os saberes fundamentais para a escolha desta profissão e $40 \%(n=2)$ disseram ainda terem sentindo-se influenciados por parentes, como mãe, irmã, que já atuavam na comercialização de tais produtos. Um dos sujeitos entrevistados mencionou que realizou um curso de capacitação sobre o uso de plantas medicinais, a fim de ampliar seu conhecimento a respeito destes vegetais, de modo a se tornar mais preparada em relação às demandas concorrentes do trabalho.

Quanto às formas de obtenção das plantas, a maior parte dos erveiros afirmou que as compravam de terceiros, sendo dependentes de produtores rurais do próprio município e de outros circunvizinhos ou ainda de atravessadores para adquiri-los. Por outro lado, um dos vendedores relatou cultivar algumas espécies vegetais em razão de trabalhar com plantas in natura. Estudos realizados por Melo, Amorim e Albuquerque (2009), Rocha et al. (2013) e Lima, Nascimento e Silva (2016), constataram também que a maioria dos entrevistados em suas pesquisas adquirem as plantas por terceiros, não realizando o cultivo.

Através das informações prestadas pelos feirantes, foi possível catalogar 59 etnoespécies, pertencentes a 55 gêneros e distribuídas em 38 famílias botânicas (Tabela 1). 
Tabela 1. Plantas medicinais encontradas no levantamento etnobotânico realizado no Mercado público, de Iguatu, Ceará, Brasil.

\begin{tabular}{|c|c|c|c|c|c|}
\hline Nome científico & Etnoespécie & Família & $\begin{array}{l}\text { Forma de } \\
\text { venda }\end{array}$ & $\begin{array}{c}\text { Forma } \\
\text { de preparo }\end{array}$ & Ação terapêutica \\
\hline $\begin{array}{c}\text { Achyrocline } \\
\text { satureioides (Lam.) } \\
\text { DC. }\end{array}$ & Marcela & Asteraceae & Semente & Chá & Dor de barriga \\
\hline Allium сера $\mathrm{L}$. & $\begin{array}{r}\text { Cebola } \\
\text { medicinal }\end{array}$ & Amaryllidaceae & Fruto & Chá & Tosse e gripe \\
\hline $\begin{array}{l}\text { Anacardium } \\
\text { occidentale } \mathrm{L} .\end{array}$ & Cajueiro & Anacardiaceae & Caule & Chá & $\begin{array}{l}\text { Diabetes e } \\
\text { inflamação }\end{array}$ \\
\hline $\begin{array}{c}\text { Anastatica } \\
\text { hierochuntica } \mathrm{L} .\end{array}$ & Jericó & Brassicaceae & $\begin{array}{l}\text { Planta } \\
\text { inteira }\end{array}$ & Chá & Gripe \\
\hline $\begin{array}{c}\text { Anethum graveolens } \\
\text { L. }\end{array}$ & Endro & Apiaceae & Semente & Chá & Insônia \\
\hline Annona muricata L. & Graviola & Annonaceae & Folha & Chá & Diabetes \\
\hline $\begin{array}{l}\text { Aristolochia labiata } \\
\text { Willd. }\end{array}$ & $\begin{array}{l}\text { Cipó mil } \\
\text { homens }\end{array}$ & Aristolochiaceae & Caule & Chá & Afrodisíaco \\
\hline $\begin{array}{l}\text { Aristolochia } \\
\text { trilobata L. }\end{array}$ & Jarrinha & Aristolochiaceae & Raiz & Chá & Abortivo \\
\hline $\begin{array}{l}\text { Baccharis trimera } \\
\text { (Less.) DC. }\end{array}$ & Carqueja & Asteraceae & Caule & Chá & $\begin{array}{l}\text { Dor na coluna e } \\
\text { emagrecimento }\end{array}$ \\
\hline $\begin{array}{l}\text { Bauhinia forficata } \\
\text { Link }\end{array}$ & Pata de vaca & Fabaceae & Folha & Chá & Problemas renais \\
\hline $\begin{array}{l}\text { Brassica nigra (L.) } \\
\text { K.Koch }\end{array}$ & Mostarda & Brassicaceae & Semente & Chá & Trombose \\
\hline $\begin{array}{l}\text { Caesalpinia ferrea } \\
\text { Mart. ex Tul. }\end{array}$ & Bagi de jucá & Fabaceae & Semente & Chá & $\begin{array}{l}\text { Gripe, tosse e } \\
\text { cansaço }\end{array}$ \\
\hline $\begin{array}{l}\text { Camellia sinensis } \\
\text { (L.) Kuntze }\end{array}$ & Chá preto & Theaceae & Semente & Chá & $\begin{array}{l}\text { Coração (dor ou } \\
\text { inchaço) }\end{array}$ \\
\hline $\begin{array}{l}\text { Camellia sinensis } \\
\text { (L.) Kuntze }\end{array}$ & Chá verde & Theaceae & Planta toda & Chá & Emagrecimento \\
\hline $\begin{array}{c}\text { Carapichea } \\
\text { ipecacuanha (Brot.) } \\
\text { L.Andersson }\end{array}$ & Ipecacuanha & Rubiaceae & Raiz & Chá & Gripe \\
\hline $\begin{array}{l}\text { Cardiospermum } \\
\text { corindum } \mathrm{L} .\end{array}$ & $\begin{array}{l}\text { Cipó de } \\
\text { vaqueiro }\end{array}$ & Sapindaceae & Raiz e caule & Chá & Problemas renais \\
\hline $\begin{array}{r}\text { Ceiba glaziovii } \\
\text { (Kuntze) K.Schum. }\end{array}$ & Barriguda & Malvaceae & Casca & Chá & Inflamação \\
\hline $\begin{array}{l}\text { Centaurea benedicta } \\
\text { (L.) L. }\end{array}$ & Cardo santo & Asteraceae & Semente & Chá & Asma \\
\hline
\end{tabular}




\begin{tabular}{|c|c|c|c|c|c|}
\hline $\begin{array}{l}\text { Coccoloba ovata } \\
\text { Benth. }\end{array}$ & Muçambeira & Polygonaceae & Raiz & Chá & Gripe \\
\hline $\begin{array}{l}\text { Cordia oncocalyx } \\
\text { Allemão }\end{array}$ & Pau branco & Boraginaceae & Casca & Chá & Para engravidar \\
\hline $\begin{array}{l}\text { Cuminum cyminum } \\
\text { L. }\end{array}$ & Cominho & Apiaceae & Semente & Chá & $\begin{array}{l}\text { Cólicas em } \\
\text { recém-nascidos }\end{array}$ \\
\hline $\begin{array}{l}\text { Equisetum arvense } \\
\text { L. }\end{array}$ & Cavalinha & Equisetaceae & Caule & Chá & Emagrecimento \\
\hline $\begin{array}{l}\text { Eschweilera ovata } \\
\text { (Cambess.) Mart. ex } \\
\text { Miers }\end{array}$ & Imbiriba & Lecythidaceae & Flor & Chá & $\begin{array}{l}\text { Dores, cólicas e } \\
\text { constipação } \\
\text { intestinal }\end{array}$ \\
\hline $\begin{array}{l}\text { Eucalyptus globulus } \\
\text { Labill. }\end{array}$ & Eucalipto & Myrtaceae & Folha & Chá & Febre e gripe \\
\hline $\begin{array}{c}\text { Handroanthus } \\
\text { serratifolius }(\text { Vahl }) \\
\text { S.O.Grose }\end{array}$ & Pau D'arco & Bignoniaceae & $\begin{array}{l}\text { Casca do } \\
\text { caule }\end{array}$ & Chá & Próstata \\
\hline $\begin{array}{l}\text { Helianthus annuus } \\
\text { L. }\end{array}$ & Girassol & Asteraceae & Semente & Chá & Trombose \\
\hline $\begin{array}{l}\text { Hibiscus acetosella } \\
\text { Welw. ex Hiern }\end{array}$ & Hibisco & Malvaceae & Flor & Chá & $\begin{array}{c}\text { Perder peso, } \\
\text { diabetes e colesterol }\end{array}$ \\
\hline $\begin{array}{c}\text { Hymenaea courbaril } \\
\text { L. }\end{array}$ & Jatobá & Fabaceae & Semente & $\begin{array}{l}\text { Vitamin } \\
\text { a da } \\
\text { semente }\end{array}$ & Anemia \\
\hline $\begin{array}{l}\text { Illicium verum } \\
\text { Hook.f. }\end{array}$ & $\begin{array}{l}\text { Anis } \\
\text { estrelado }\end{array}$ & Schisandraceae & Flor & Chá & Asma \\
\hline $\begin{array}{l}\text { Jacaranda caroba } \\
\text { (Vell.) DC. }\end{array}$ & Caroba & Bignoniaceae & Casca & Chá & $\begin{array}{c}\text { Pitiríase } \\
\text { versicolor (pano } \\
\text { branco) e outras } \\
\text { infecções cutâneas }\end{array}$ \\
\hline $\begin{array}{l}\text { Licania rigida } \\
\text { Benth. }\end{array}$ & Oiticica & Chrysobalanaceae & Folha & Chá & Diabetes \\
\hline $\begin{array}{l}\text { Maytenus ilicifolia } \\
\text { Mart. ex Reissek }\end{array}$ & $\begin{array}{l}\text { Espinheira } \\
\text { santa }\end{array}$ & Celastraceae & Folha & Chá & $\begin{array}{l}\text { Diabetes e } \\
\text { gastrite }\end{array}$ \\
\hline $\begin{array}{l}\text { Miconia albicans } \\
\text { (Sw.) Steud. }\end{array}$ & $\begin{array}{l}\text { Canela de } \\
\text { velho }\end{array}$ & Melastomataceae & $\begin{array}{l}\text { Planta } \\
\text { inteira }\end{array}$ & Chá & Dor na coluna \\
\hline $\begin{array}{l}\text { Moringa oleifera } \\
\text { Lam. }\end{array}$ & Moringa & Moringaceae & Folha & Chá & $\begin{array}{l}\text { Dores no geral e } \\
\text { inflamação }\end{array}$ \\
\hline $\begin{array}{l}\text { Myristica fragrans } \\
\text { Houtt }\end{array}$ & Noz moscada & Myristicaceae & Semente & Chá & $\begin{array}{c}\text { Trombose e } \\
\text { derrame cerebral }\end{array}$ \\
\hline $\begin{array}{l}\text { Ocimum basilicum } \\
\text { L. }\end{array}$ & Manjericão & Lamiaceae & Folha & Chá & Sinusite \\
\hline
\end{tabular}




\begin{tabular}{|c|c|c|c|c|c|}
\hline $\begin{array}{c}\text { Ocimum } \\
\text { gratissimum } \mathrm{L} .\end{array}$ & Alfavaca & Lamiaceae & Folha & Chá & Sinusite \\
\hline $\begin{array}{c}\text { Operculina } \\
\text { macrocarpa }(\text { L.) Urb. }\end{array}$ & $\begin{array}{l}\text { Batata de } \\
\text { purga }\end{array}$ & Convolvulaceae & Caule & Chá & $\begin{array}{l}\text { Ferimentos no } \\
\text { corpo e infecções } \\
\text { cutâneas }\end{array}$ \\
\hline $\begin{array}{l}\text { Persea americana } \\
\text { Mill. }\end{array}$ & Abacateiro & Lauraceae & Folha & Chá & Problemas renais \\
\hline Petiveria alliacea $\mathrm{L}$. & Tipí & Phytolaccaceae & Raiz & Chá & Reumatismo \\
\hline $\begin{array}{l}\text { Peumus boldus } \\
\text { Molina }\end{array}$ & $\begin{array}{l}\text { Boldo-do- } \\
\text { chile }\end{array}$ & Monimiaceae & Folha & Chá & $\begin{array}{l}\text { Problemas } \\
\text { hepáticos }\end{array}$ \\
\hline $\begin{array}{l}\text { Phyllanthus niruri } \\
\text { L. }\end{array}$ & Quebra pedra & Phyllanthaceae & Caule e raíz & Chá & Cálculos renais \\
\hline $\begin{array}{l}\text { Pimpinella anisum } \\
\text { L. }\end{array}$ & Erva doce & Apiaceae & Semente & Chá & $\begin{array}{l}\text { Tranquilizante e } \\
\text { normaliza o } \\
\text { estômago }\end{array}$ \\
\hline $\begin{array}{c}\text { Pterodon } \\
\text { emarginatus Vogel }\end{array}$ & Sucupira & Fabaceae & Semente & Chá & Dores na coluna \\
\hline Punica granatum L. & Romã & Lythraceae & $\begin{array}{l}\text { Casca e } \\
\text { semente }\end{array}$ & Chá & $\begin{array}{l}\text { Garganta e anti- } \\
\text { inflamatório }\end{array}$ \\
\hline $\begin{array}{l}\text { Rosmarinus } \\
\text { officinalis L. }\end{array}$ & $\begin{array}{l}\text { Alecrim do } \\
\text { reino }\end{array}$ & Lamiaceae & Folha & Chá & Sinusite \\
\hline Scoparia dulcis $L$. & Vassourinha & Plantaginaceae & Raiz & Chá & Infecção urinária \\
\hline $\begin{array}{l}\text { Senna alexandrina } \\
\text { Mil }\end{array}$ & Sene & Fabaceae & Folha & Chá & $\begin{array}{r}\text { Problemas no } \\
\text { ciclo Menstrual }\end{array}$ \\
\hline $\begin{array}{c}\text { Sesamum } \\
\text { abbreviatum Merxm. }\end{array}$ & $\begin{array}{l}\text { Girgilim } \\
\text { amarelo }\end{array}$ & Pedaliaceae & Semente & Chá & $\begin{array}{c}\text { Ajuda nas } \\
\text { contrações durante } \\
\text { o parto }\end{array}$ \\
\hline Sesamum indicum $\mathrm{L}$. & $\begin{array}{l}\text { Gergelim } \\
\text { preto }\end{array}$ & Pedaliaceae & Semente & Chá & Trombose facial \\
\hline $\begin{array}{c}\text { Sideroxylon } \\
\text { obtusifolium (Roem. \& } \\
\text { Schult.) T.D.Penn. }\end{array}$ & Quixabeira & Sapotaceae & Casca & Chá & Câncer \\
\hline $\begin{array}{c}\text { Solanum } \\
\text { paniculatum } \mathrm{L} .\end{array}$ & $\begin{array}{l}\text { Jurubeba } \\
\text { branca }\end{array}$ & Solanaceae & Raiz & Chá & $\begin{array}{l}\text { Infecções } \\
\text { cutâneas }\end{array}$ \\
\hline $\begin{array}{l}\text { Trichilia catigua } \\
\text { A.Juss. }\end{array}$ & Catuaba & Meliaceae & Casca & Chá & $\begin{array}{c}\text { Impotência } \\
\text { sexual masculina }\end{array}$ \\
\hline Turnera ulmifolia $\mathrm{L}$. & Chanana & Turneraceae & Raiz & Chá & Inflamação \\
\hline $\begin{array}{r}\text { Uncaria tomentosa } \\
\text { (Willd. ex Schult.) DC. }\end{array}$ & Unha de gato & Rubiaceae & Casca & Chá & Inflamação \\
\hline $\begin{array}{l}\text { Vitex gardneriana } \\
\text { Schauer }\end{array}$ & Jaramataia & Lamiaceae & Folha & Chá & Inflamação \\
\hline
\end{tabular}




\begin{tabular}{|c|c|c|c|c|c|}
\hline $\begin{array}{c}\text { Ximenia americana } \\
\text { L. }\end{array}$ & $\begin{array}{l}\text { Ameixa do } \\
\text { mato }\end{array}$ & Olacaceae & Casca & Chá & Inflamação \\
\hline $\begin{array}{l}\text { Xylopia aromatica } \\
\text { (Lam.) Mart. }\end{array}$ & $\begin{array}{l}\text { Pimenta de } \\
\text { macaco }\end{array}$ & Annonaceae & Planta toda & Chá & Dores em geral \\
\hline $\begin{array}{l}\text { Zingiber officinale } \\
\text { Roscoe }\end{array}$ & Gengibre & Zingiberaceae & $\begin{array}{l}\text { Caule } \\
\text { rizoma }\end{array}$ & Chá & $\begin{array}{l}\text { Dor na garganta, } \\
\text { congestão nasal e } \\
\text { emagrecimento }\end{array}$ \\
\hline
\end{tabular}

Fonte: Autores.

As famílias botânicas mais bem representadas, com o maior número de espécies citadas, foram Fabaceae (5 espécies), Asteraceae e Lamiaceae (4 espécies cada) e Apiaceae (3 espécies) (Figura 1). Essas famílias juntas correspondem a 27,1\% do total de espécies catalogadas.

Resultados semelhantes foram encontrados por Alves et al. (2016) em levantamento etnobotânico de plantas medicinais comercializadas pelos raizeiros da feira livre do município de Guarabira no estado da Paraíba, no qual se verificou que a maioria das espécies investigadas pertencem às famílias Fabaceae, Lamiaceae e Asteraceae. Lós, Barros e Neves (2012), relata que as famílias Fabaceae e Lamiaceae, bem como Asteraceae, obtiveram uma maior representatividade em sua pesquisa, salientando-se a importância cultural delas, por serem amplamente distribuídas em regiões temperadas e tropicais do mundo.

Fabaceae está entre as famílias de maior diversidade, tendo muitas de suas espécies comercializadas em mercados e feiras para fins medicinais (Alves et al., 2007) e, ainda, é comum o uso delas em várias comunidades rurais da Caatinga (Loiola et al., 2010; Silva et al., 2015). A família Lamiaceae, composta majoritariamente por herbáceas, é fonte natural de metabólitos bioativos, com comprovada ação farmacológica e potencial biotecnológico, como os princípios ativos presentes em óleos essenciais (Karpiński, 2020; Nieto, 2017).

Figura 1. Famílias botânicas com maior número de espécies catalogadas em Iguatu, Ceará.

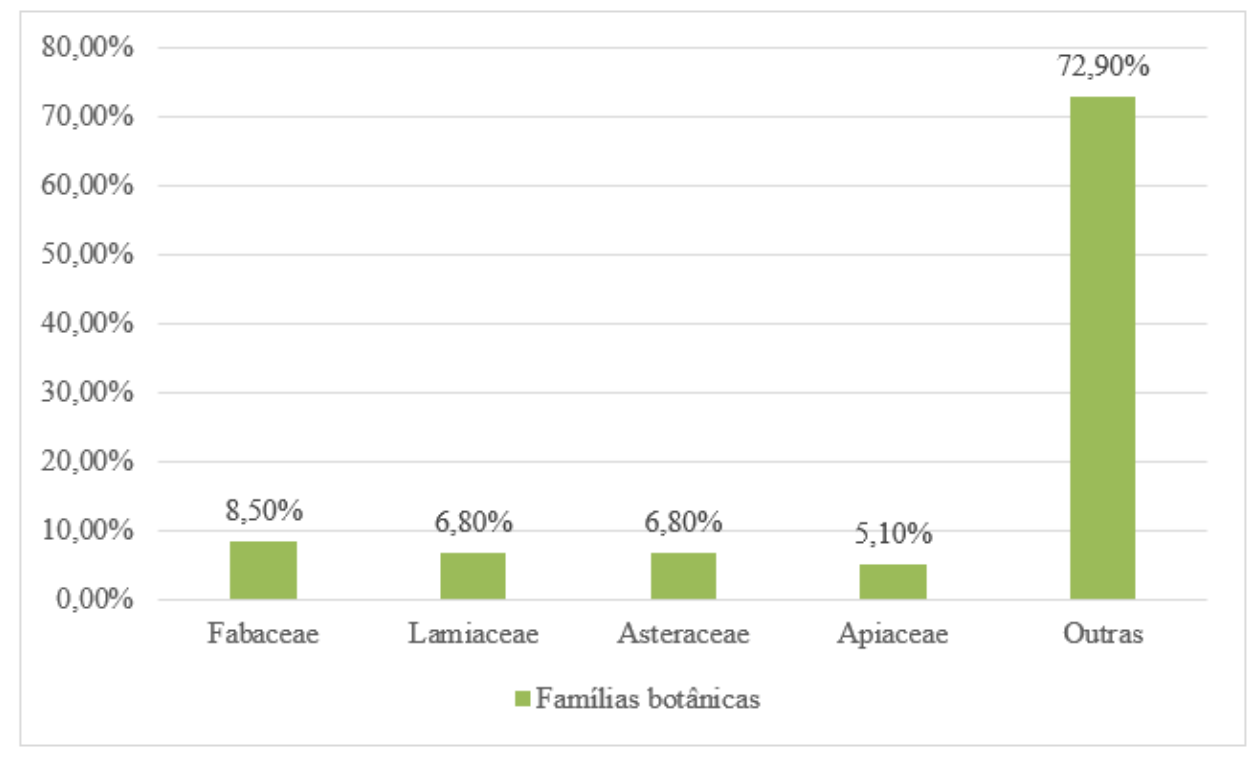

Fonte: Autores.

Outra família com grande representatividade é Asteraceae, a qual apresenta distribuição cosmopolita, sendo considerada a maior dentro do grupo das Eudicotiledôneas, com 1600-1700 gêneros e 24.000-30.000 espécies (Stevens, 2017). 
Segundo Bessada et al. (2015) e Sobrinho et al. (2017), várias espécies dessa família possuem atividade antimicrobiana, analgésica, antioxidante e anti-inflamatória comprovadas.

Quanto às partes vegetais indicadas nas preparações dos remédios caseiros, observou-se que as cascas ou caules (Figura 2) representam os recursos vegetais mais utilizados (27,4\%), seguidos pelas sementes $(24,2 \%)$, folhas (20,1\%), raízes $(14,5 \%)$, planta inteira $(6,4 \%)$, flores $(4,9 \%)$ e frutos $(1,6 \%)$. Algumas das plantas catalogadas: jericó (A. hierochuntica); canela de velho (M. albicans); quebra pedra ( $P$. niruri); chá verde (C. sinensis); romã ( $P$. granatum); pimenta de macaco $(X$. aromatica); e cipó de vaqueiro (C. corindum), apresentam mais de um recurso sendo vendido no mercado público de Iguatu.

As cascas/caules também corresponderam aos recursos mais comercializados nos estudos de Almeida e Albuquerque (2002), na feira de Caruaru no estado de Pernambuco; de Bitu et al. (2015), ocorrido nos mercados públicos do triangulo Crajubar, englobando os municípios Crato, Juazeiro do Norte e Barbalha, no estado do Ceará; e de Lima, Coelho-Ferreira e Oliveira (2011), realizado nas feiras e mercados públicos do Distrito Florestal Sustentável da BR-163 no estado do Pará. No entanto, as partes mais usadas podem oscilar conforme a região (Albuquerque et al., 2007), sendo a diferença na concentração de princípios ativos de um órgão para outro, a disponibilidade das partes das plantas e a facilidade de coleta fatores que exercem influência nesse sentido (Coelho-Ferreira, 2009).

O modo de preparo predominante das plantas medicinais informadas foi o chá, sendo empregado no preparo de 58 espécies $(98,3 \%)$. A prevalência do chá entre as formas de preparo foi encontrada também nos resultados de estudos desenvolvidos em feiras livres e mercados públicos em regiões brasileiras dos municípios da Paraíba, tais como Patos (Medeiros et al., 2019) e Esperança (Souza, Andrade, Fernandes, 2011) e de Mato Grosso, em Cuiabá (Pinto et al., 2013). A utilização de chás caseiros é uma prática associada ao conhecimento popular, geralmente repassada entre as gerações, estando, dessa maneira, relacionada às tradições e costumes socioculturais (Siqueira et al., 2006).

Figura 2. Partes vegetais comercializadas para fins medicinais em Iguatu, Ceará.

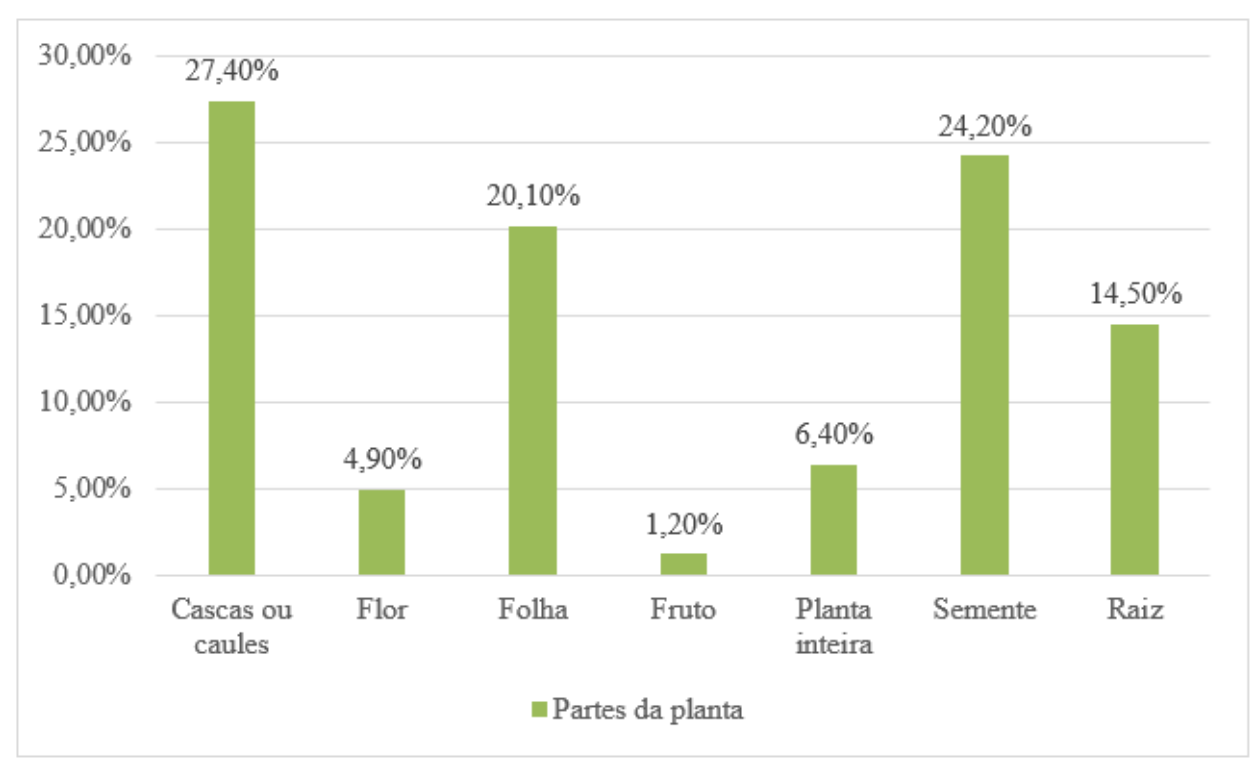

Fonte: Autores.

Os chás podem ser obtidos por infusão ou decocção (Pedro et al., 2016), sendo essa variação dependente da parte do vegetal a ser empregada, como constatado por Vásquez, Mendonça e Noda (2014) ao realizar um levantamento etnobotânico das plantas medicinais utilizadas em quatro comunidades ribeirinhas do município de Manacapuru no Amazonas. Os autores salientam que a infusão é indicada para quando as partes mais tenras das plantas são usadas, tais como folhas, flores, 
inflorescências e frutos, não havendo a necessidade de ferver a planta, enquanto a decocção é feita com as partes menos maleáveis dos vegetais, como cascas, raízes, sementes e caules.

Dentre as 59 etnoespécies listadas, 13 (22\%) são utilizadas para tratar mais de um problema de saúde, enquanto 46 (78\%) possuem apenas uma indicação terapêutica. As ações medicinais mais comuns dos vegetais relatados foram para o tratamento de enfermidades, como: gripes, inflamações, problemas nos rins e diabetes, assemelhando-se aos resultados encontrados na pesquisa de Lima, Nascimento e Silva (2016) a qual verificou o comércio de plantas medicinais nas feiras livres do município de Arapiraca, no estado de Alagoas. Dentre as plantas medicinais utilizadas para o tratamento das citadas doenças, respectivamente, ipecacuanha (C. ipecacuanha), unha de gato (U. tomentosa), pata de vaca (B. forficata) e espinheira santa (M. ilicifolia).

No que concerne à exposição e ao armazenamento das plantas medicinais nos boxes da feira livre e do mercado público de Iguatu, constatou-se, excetuando a banca de um feirante que trabalha com ervas in natura, inadequado manuseio de modo a comprometer a higiene e a conservação. Em decorrência das bancadas e prateleiras dos boxes apresentarem um tamanho pequeno, a maior parte das plantas ficam aglomeradas, não havendo separação física entre os produtos, enquanto algumas são embaladas em jornais ou em sacos plásticos. Estas condições precárias de armazenamento das plantas, secas ou in natura, favorecem o desenvolvimento de micro-organismos oportunistas e patogênicos, como fungos. Tais condições sanitárias podem ocasionar casos de intoxicação, como relatado no estudo de Lanini et al. (2009), em que um raizeiro do município de Diadema, no estado de São Paulo, relatou um caso de intoxicação de uma cliente ao ingerir chás feitos com folhas contaminadas por fungos.

Esses problemas condizem com os achados em pesquisa similar realizada por Bispo et al. (2019), em dois mercados tradicionais na cidade de Juazeiro do Norte, Ceará, os quais observaram que a maior parte dos estabelecimentos visitados não possui local apropriado para o armazenamento das ervas desidratadas, havendo falta de organização, separação e identificação do material.

A Relação Nacional de Plantas Medicinais de Interesse ao SUS (RENISUS) compreende uma listagem oficial de espécies vegetais com potencial para gerar produtos de interesse ao SUS, sendo composta por 71 espécies (Brasil, 2009). Do total de 59 plantas medicinais comercializadas no Mercado Municipal de Iguatu, 12 estão incluídas na RENISUS (A. occidentale, B. trimera, B. forficata, C. ferrea, E. arvense, E. globulus, O. gratissimum, P. americana, P. granatum, S. paniculatum, U. tomentosa, Z. officinale). Este dado corrobora com a importância etnomedicinal e biotecnológica que essas espécies possuem. Outrossim, são espécies em sua maioria precursoras de produtos ou derivados com finalidade diversa para a indústria farmacêutica, alimentícia e estética.

\section{Considerações Finais}

A realização desta pesquisa permitiu identificar uma variedade de espécies de plantas medicinais comercializadas em uma feira livre e no Mercado Público Municipal de Iguatu, Ceará. O estudo destacou as partes utilizadas das ervas e seus efeitos medicinais conhecidos pelos feirantes da região. Em relação às famílias botânicas catalogadas, destacam-se, nesta sequência, Fabaceae, Lamiaceae, Asteraceae e Apiaceae. Diante da pesquisa realizada com os feirantes, observou que eles não dispunham de um espaço adequado para comercializar ou armazenar suas mercadorias, com condições inadequadas de higiene e manuseio dos produtos vegetais, expondo assim as ervas medicinais a vários micro-organismos potencialmente infecciosos.

De acordo com as características do sistema de produção de plantas medicinais, a maioria dos erveiros afirmou desconhecer o procedimento do cultivo e a fonte de origem das ervas comercializadas. Todos os entrevistados relataram que os conhecimentos obtidos haviam sido repassados por seus ancestrais, comprovando o caráter empírico e a importância cultural e econômica de comercializar plantas medicinais em feiras livres. Além disso, este relato científico auxilia na valorização e 
comunicação dos conhecimentos tradicionais, de grande relevância para a população.

\section{Agradecimentos}

Os autores agradecem aos sujeitos da pesquisa, que se disponibilizaram em participar do estudo, o qual também envolveu ações de extensão universitária. Também agradecem ao apoio de fomento da Fundação Cearense de Apoio ao Desenvolvimento Científico e Tecnológico-FUNCAP, bem como ao projeto de extensão universitária "Uso e comercialização de plantas medicinais em feiras livres e mercados públicos de Iguatu-Ceará, construindo saberes e identificando práticas sociais" do curso de Ciências Biológicas da Faculdade de Educação, Ciências e Letras de Iguatu, unidade da Universidade Estadual do Ceará.

\section{Referências}

Albuquerque, U. P., da Cunha, L. V. F. C., De Lucena, R. F. P., \& Alves, R. R. N. (Eds.). (2014). Methods and techniques in ethnobiology and ethnoecology. Humana Press.

Albuquerque, U. D., Lucena, R. D., \& Cunha, L. V. F. C. (2004). Métodos e técnicas na pesquisa etnobotânica. Livro Rápido/NUPEEA, 19.

Albuquerque, U. P., Monteiro, J. M., Ramos, M. A., \& de Amorim, E. L. C. (2007). Medicinal and magic plants from a public market in northeastern Brazil. Journal of ethnopharmacology, 110(1), 76-91.

Almeida, C. D. F. C. B., \& Albuquerque, U. P. (2002). Uso e conservação de plantas e animais medicinais no estado de Pernambuco (Nordeste do Brasil): um estudo de caso. Interciencia, 27(6), 276-285.

Alves, C. A. B., Silva, S., Belarmino, N. A. L. A., Souza, R. S., Silva, D. R., Alves, P. R. R., \& Nunes, G. M. (2016). Comercialização de plantas medicinais: um estudo etnobotânico na feira livre do município de Guarabira, Paraíba, nordeste do Brasil. Gaia Scientia, 10(4), $390-407$.

Alves, R. R. D. N., Silva, A. A. G., Souto, W. M. S., \& Barboza, R. R. D. (2007). Utilização e comércio de plantas medicinais em Campina Grande, PB, Brasil. Revista eletrônica de farmácia, 4(2).

Azevedo, S. K. S. D., \& Silva, I. M. (2006). Plantas medicinais e de uso religioso comercializadas em mercados e feiras livres no Rio de Janeiro, RJ, Brasil. Acta botânica brasilica, 20(1), 185-194.

Barbosa, O. Economia popular Feira livre de Iguatu mantém tradição e atrai consumidores. Diário do Nordeste, Fortaleza, (2019). http://blogs.diariodonordeste.com.br/centrosul/cidades/feira-livre-de-iguatu-mantem-tradicao-e-atrai-consumidores-aos-sabados/31202.

Barreto, M. R., \& Spanholi, M. L. (2019). Estudio etnobotánico en comunidades rurales de Sinop, Mato Grosso, Brasil. Interações (Campo Grande), 20(1), 267-282.

Bessada, S. M., Barreira, J. C., \& Oliveira, M. B. P. (2015). Asteraceae species with most prominent bioactivity and their potential applications: A review. Industrial Crops and Products, 76, 604-615.

Bispo, G. L., Marco, C. A., Silva Ferreira, F., Chaves, J. T. L., \& Farias, R. B. (2019). Estudo etnobotânico de plantas medicinais no comércio da cidade de Juazeiro do Norte, CE. Journal of Biology \& Pharmacy and Agricultural Management, 15(4).

Bitu, V. D. C. N., Bitu, V. D. C. N., Matias, E. F. F., Lima, W. P., Costa Portelo, A., Coutinho, H. D. M., \& de Menezes, I. R. A. (2015). Ethnopharmacological study of plants sold for therapeutic purposes in public markets in Northeast Brazil. Journal of Ethnopharmacology, 172, $265-272$.

Bochner, R., Fiszon, J. T., Assis, M. A., \& Avelar, K. E. S. (2012). Problemas associados ao uso de plantas medicinais comercializadas no Mercadão de Madureira, município do Rio de Janeiro, Brasil. Revista Brasileira de Plantas Medicinais, 14(3), 537-547.

Brasil. (2009). Ministério da Saúde. RENISUS - Relação Nacional de Plantas Medicinais de Interesse ao SUS. Espécies vegetais. DAF/SCTIE/MS RENISUS. http://portal.saude.gov.br/portal/ arquivos/pdf/RENISUS.pdf.

Brasil. (2006a). Ministério da Saúde. Secretaria de Atenção à Saúde. Departamento de Atenção Básica. Política Nacional de Práticas Integrativas e Complementares no SUS - PNPIC-SUS. Brasília: Ministério da Saúde, 92 p.

Brasil. (2006a). Ministério da Saúde. Secretaria de Ciência, Tecnologia e Insumos Estratégicos. Departamento de Assistência Farmacêutica. Política nacional de plantas medicinais e fitoterápicos. Brasília: Ministério da Saúde, 60 p.

Cerqueira, T. M. G., Correia, A. C. D. C., Santos, R. V. D., Lemos, R. P. L., Silva, S. A. S. D., \& Barreto, E. (2020). The Use of Medicinal Plants in Maceió, Northeastern Brazil: An Ethnobotanical Survey. Medicines, 7(2), 7.

Coelho-Ferreira, M. (2009). Medicinal knowledge and plant utilization in an Amazonian coastal community of Marudá, Pará State (Brazil). Journal of Ethnopharmacology, 126(1), 159-175.

Couto, X. C. S. (2016). Cultivando agricultores: Percepção dos jovens rurais sobre as plantas medicinais. Cadernos de Agroecologia, 10(3). 
David, M., \& Pasa, M. C. (2015). As plantas medicinais e a etnobotânica em Várzea Grande, MT, Brasil. Interações (Campo Grande), 16(1), 97-108.

Freitas, A. V. L., Coelho, M. D. F. B., de Azevedo, R. A. B., \& Maia, S. S. S. (2012). Os raizeiros e a comercialização de plantas medicinais em São Miguel, Rio Grande do Norte, Brasil. Revista Brasileira de Biociências, 10(2), 147.

IBGE - Instituto Brasileiro de Geografia e Estatística. (2020a). Panorama: Ceará. https://cidades.ibge.gov.br/brasil/ce/panorama.

IBGE - Instituto Brasileiro de Geografia e Estatística. (2020b). Panorama: Iguatu - Ceará. http://cod.ibge.gov.br/28SF.

IPECE - Instituto de Pesquisa e Estratégia Econômica do Ceará. (2017). Perfil Básico do Município de Iguatu. https://www.ipece.ce.gov.br/perfil-municipal$2017 /$.

Karpiński, T. M. (2020). Essential oils of Lamiaceae family plants as antifungals. Biomolecules, 10(1), 103.

Lanini, J., Duarte-Almeida, J. M., Nappo, S., \& Carlini, E. A. (2009). " O que vêm da terra não faz mal": relatos de problemas relacionados ao uso de plantas medicinais por raizeiros de Diadema/SP. Revista Brasileira de Farmacognosia, 19(1A), 121-129.

Lima, I. E. O., Nascimento, L. A. M., \& Silva, M. S. (2016). Comercialização de plantas medicinais no município de Arapiraca-AL. Revista Brasileira de Plantas Medicinais, 18(2), 462-472.

Lima, P. G. C., Coelho-Ferreira, M., \& Oliveira, R. (2011). Plantas medicinais em feiras e mercados públicos do Distrito Florestal Sustentável da BR-163, estado do Pará, Brasil. Acta Botanica Brasilica, 25(2), 422-434.

Loch, V. C., Saraiva, R. V. C., Souza, R., Barroso, K., da Rocha Sobrinho, M., \& Silva, H. R. (2020). Os raizeiros e as plantas medicinais comercializadas nas feiras livres de São Luís, MA. Cadernos de Agroecologia, 15(2).

Loiola, M. I. B., de Carvalho Paterno, G. B., Diniz, J. A., Calado, J. F., \& Oliveira, A. C. P. (2010). Leguminosas e seu potencial de uso em comunidades rurais de São Miguel do Gostoso-RN. Revista Caatinga, 23(3), 59-70.

Lós, D. W. S., Barros, R. P., \& Neves, J. D. S. (2012). Comercialização de plantas medicinais: um estudo etnobotânico nas feiras livres do município de Arapiraca-AL. Biofar, 7(2), 38-51.

Maioli-Azevedo, V., \& Fonseca-Kruel, V. S. D. (2007). Plantas medicinais e ritualísticas vendidas em feiras livres no Município do Rio de Janeiro, RJ, Brasil: estudo de caso nas zonas Norte e Sul. Acta Botanica Brasilica, 21(2), 263-275.

Medeiros, F. S., Sá, G. B., Dantas, M. K. L., \& Gracias Almeida, M. (2019). Plantas medicinais comercializadas na feira livre do município de Patos, Paraíba. Revista Verde de Agroecologia e Desenvolvimento Sustentável, 14(1), 150-155.

Melo, J. G., Amorim, E. L. C., \& Albuquerque, U. P. (2009). Native medicinal plants commercialized in Brazil-priorities for conservation. Environmental Monitoring and Assessment, 156(1), 567-580.

Nieto, G. (2017). Biological activities of three essential oils of the Lamiaceae family. Medicines, 4(3), 63.

Novaes, T. E. R., Novaes, A. S. R., Glusczak, L., \& Vilarinho, L. B. O. (2021). Potenciais medicinais da faveleira (Cnidoscolus quercifolius) e seus usos na saúde humana: uma breve revisão. Research, Society and Development, 10(2), e43910212845.

Pedro, F. G. G., Arruda, G. L., Oliveira, J. C., Santos, A. D., Sigarini, K., Hernandes, T., Villa, R. D., \& Oliveira, A. P. (2016). Composição centesimal e mineral de plantas medicinais comercializadas no mercado do Porto de Cuiabá, Mato Grosso, Brasil. Revista Brasileira de Plantas Medicinais, 18(1), 297-306.

Pinto, A. Z. D. L., Assis, A. F. S., Pereira, A. G., \& Pasa, M. C. (2013). Levantamento etnobotânico de plantas medicinais comercializadas no mercado do Porto em Cuiabá, Mato Grosso, Brasil. FLOVET-Boletim do Grupo de Pesquisa da Flora, Vegetação e Etnobotânica, 1(5).

Rocha, F. A. G., Araújo, M. F. F., Costa, N. D. L., Silva, R. P., Queiroga, P. V. D. M., Marciano, L. A., Pontes, E. D. M., \& Souza, J. A. B. (2013). Características socioeconômicas dos comerciantes de plantas medicinais de Currais Novos/RN. Holos, 4, 87-100.

Sales, A. P., Rezende, L. T., \& Sette, R. D. S. (2011). Negócio feira livre: um estudo em um município de Minas Gerais. III Encontro de Gestão de Pessoas e Relações de Trabalho, ANPAD, Anais..., João Pessoa/PB.

Santos, J. J. F., Coelho-Ferreira, M., \& Lima, P. G. C. (2018). Etnobotânica de plantas medicinais em mercados públicos da Região Metropolitana de Belém do Pará, Brasil. Biota Amazônia (Biote Amazonie, Biota Amazonia, Amazonian Biota), 8(1), 1-9.

Santos, M., Vieira, I. R., Sailva, M., \& Andrade, I. D. (2019). Comercialização de plantas medicinais nos mercados públicos do município de Parnaíba, Piauí, Brasil. Espacios, 40(22), 1-13.

Silva, C. G., Marinho, M. G. V., Lucena, M. F. A., \& Costa, J. G. M. (2015). Levantamento etnobotânico de plantas medicinais em área de Caatinga na comunidade do Sítio Nazaré, município de Milagres, Ceará, Brasil. Revista Brasileira de Plantas Medicinais, 17(1), $133-142$.

Siqueira, K. M., Barbosa, M. A., Brasil, V. V., Oliveira, L. M. C., \& Andraus, L. M. S. (2006). Crenças populares referentes à saúde: apropriação de saberes sócio-culturais. Texto \& Contexto-Enfermagem, 15(1), 68-73.

Sobrinho, A. C. N., Morais, S. M., Souza, E. B., \& Fontenelle, R. O. S. (2017). The genus Eupatorium L. (Asteraceae): A review of their antimicrobial activity. Journal of Medicinal Plants Research, 11(3), 43-57.

Souza, M. Z. S., Andrade, L. R. S., \& Fernandes, M. S. M. (2011). Levantamento sobre plantas medicinais comercializadas na feira livre da cidade de Esperança-PB. Revista de Biologia e Farmácia, 5(1), 111-118.

Stevens, P. F. (2017). Angiosperm Phylogeny Website. Version 14. http://www.mobot.org/MOBOT/research/APweb.. 
Research, Society and Development, v. 10, n. 6, e14310615478, 2021

(CC BY 4.0) | ISSN 2525-3409 | DOI: http://dx.doi.org/10.33448/rsd-v10i6.15478

Vásquez, S. P. F., Mendonça, M. S. D., \& Noda, S. D. N. (2014). Etnobotânica de plantas medicinais em comunidades ribeirinhas do Município de Manacapuru, Amazonas, Brasil. Acta Amazonica, 44(4), 457-472.

Williams, V. L., Witkowski, E. T., \& Balkwill, K. (2005). Application of diversity indices to appraise plant availability in the traditional medicinal markets of Johannesburg, South Africa. Biodiversity \& Conservation, 14(12), 2971-3001. 